\title{
Optimal Resource Allocation and Task Segmentation in loT Enabled Mobile Edge Cloud
}

This paper was downloaded from TechRxiv (https://www.techrxiv.org).

\section{LICENSE}

CC BY 4.0

SUBMISSION DATE / POSTED DATE

02-10-2021 / 07-10-2021

\section{CITATION}

Mahmood, Asad; Hong, Yue; Khurram Ehsan, Muhammad; Mumtaz, Shahid (2021): Optimal Resource Allocation and Task Segmentation in loT Enabled Mobile Edge Cloud. TechRxiv. Preprint. https://doi.org/10.36227/techrxiv.16726561.v1

$\mathrm{DOI}$ 


\title{
Optimal Resource Allocation and Task Segmentation in IoT Enabled Mobile Edge Cloud
}

\author{
Asad Mahmood, Yue Hong, Muhammad Khurram Ehsan and Shahid Mumtaz, Senior Member, IEEE
}

\begin{abstract}
1 Abstract-Recent development toward innovative applications and technologies like self-driving, augmented reality, smart cities, and various other applications leads to excessive growth in the number of devices. These devices have finite computation resources and cannot handle the applications that require extensive computation with minimal delay. To overcome this, the mobile edge cloud (MEC) emerges as a practical solution that allows devices to offload their extensive computation to MEC located in their vicinity; this will lead to succeeding the arduous delay of the millisecond scale: requirement of $5^{t h}$ generation communication system. This work examines the convex optimization problem. The objective is to minimize the task duration by optimal allocation of the resources like local and edge computational capabilities, transmission power, and optimal task segmentation. For optimal allocation of resources, an algorithm name Estimation of Optimal Resource Allocator (EORA) is designed to optimize the function by keeping track of statistics of each candidate of the population. Using EORA, a comparative analysis of the hybrid approach (partial offloading) and edge computation only is performed. Results reveal the fundamental trade-off between both of these models. Simultaneously, the impact of devices' computational capability, data volume, and computational cycles requirement on task segmentation is analyzed. Simulation results demonstrate that the hybrid approach: partial offloading scheme reduces the task's computation time and outperforms edge computing only.
\end{abstract}

Index Terms-Mobile Edge Cloud Computing, Partial Offloading Scheme, Resource Allocation

\section{INTRODUCTION}

\section{A. Background and Related Work}

The recent development in communication and computing systems leads to a dramatic revolution in the world [1]. Simultaneously, the evolution in cloud computing and the growth of the Internet of Things (IoTs) can play a vital role in enhancing users' quality of service [2]-[6]. The integration of information technology (IT), IoTs, and cloud computing lead toward the concept of smart cities, where advanced sensors are used to manage the assents [7]-[9]. From the past few decades, the popularity of devices such as smartphones, tablets, and

Asad Mahmood is with the the College of Mechatronics and Control Engineering, Shenzhen University, Shenzhen 518060, China \& also with Interdisciplinary Center for Security, Reliability and Trust (SnT), University of Luxembourg, 4365 Esch-sur-Alzette, Luxembourg (email: asad10125@ hotmail.com, asad.mahmood@uni.lu)

Yue Hong is with the College of Mechatronics and Control Engineering, Shenzhen University, Shenzhen 518060, China (email: yhong210@ gmail.com)

Muhammad Khurram Ehsan is with the Faculty of Engineering, Bahria University, Lahore Campus, 54600, Pakistan (email: mehsan.bulc@bahria.edu.pk)

Shahid Mumtaz is with the IT - Aveiro Instituto de Telecomunicações P3810-193 AVEIRO - PORTUGAL (e-mail: smumtaz@av.it.pt).

1 ,'This work is supported by funding Natural Science Foundation of SZU (grant no. 2110271)" portable devices has been accelerating toward developing the internet of things (IoTs) [10], [11]. New mobile applications such as face recognition, natural language processing, and augmented reality gain much attention from modern eras' mobile users. Besides, in the smart city, a large volume of data is collected in real-time by the devices. These intelligent gadgets are utilized in monitoring, traffic and pollution control, health facility, lighting, and other security aspects [12], [13]. On the other hand, these devices' computational capabilities are not enough to handle the applications with massive processing requirements in a short time [7], [14]. Central clouds are deployed to meets resources hungry applications' requirements, having rich resources like computational capabilities, storage, and communication infrastructure. Besides, these clouds are too far away from end-users [15], [16]. It is not adequate to rely on the central cloud. Because in the $5^{\text {th }}$ generation of communication systems, a millisecond of delay plays its vital role in the wireless communication system [17]-[19].

To meet the requirement of resource-hungry application or to meet the quality of services (QoS) requirement of endusers, a new paradigm technology known as mobile edge cloud (MEC) emerges as an effective technique to overcome the desired need [13], [20]-[23]. MEC deployed at the access point (AP), having unlimited resources which are utilized to compute end-users' task [24]. Mobile users offload their tasks to MEC in their vicinity either by using partial offloading or binary offloading. In the partial offloading scheme task is divided into two parts. One portion of the job is to compute locally using their computational resources. At the same time, the other part is overwhelmed at MEC for further processing. In contrast, in a binary offloading scheme task as a whole is computed locally or placed at the MEC for further processing [24].

MEC offers several advantages compared to the central cloud. The main advantage of MEC is the reduction in time duration. It constitutes transmission time: to place the task at MEC, execution time at MEC, and time taken by the cloud to send the task results back to users. At the same time, a reduction in the energy consumption of devices is another advantage of MEC [15]. In [25], the author proposed that delay-sensitive tasks should compute at the cloudlets; in contrast, place the other task at the central cloud for computation. The author in [26] states that if the task has a large volume of data, it requires minimal computation, so it is better to compute it locally than the task that required high computations. The author in [27] proposed a task offloading scenario, whether the task is to be placed at MEC or in the cloud-based upon its mobility. In contrast, the objective is to 
minimize energy consumption. Whereas task offloaded to the cloud by cellular network will result in a considerable delay in the system.

In recent days, task offloading strategy and optimal resource allocation among devices and MEC gain much attention from the researcher to overcome the existing system's limitations, which increases QoS or performance of the system. In [28], the author proposed the architecture of edge computing and the proposed algorithm for efficient offloading results to minimize the task's completion time. In [29], the author focused on ultra-dense networks and proposed a mobility management scheme by considering a user-centric approach for mobile edge computing. According to the author in [30], MEC reduces the latency and saves energy by optimizing resources and task placement. The author in [24] considers both optimal allocations of resources and offloading schemes to increase devices' energy efficiency. In [31], [32], the author proposed the software Define Ultra-Dense Network architecture to minimize the latency by efficiently offloading tasks to MEC using a binary offloading scheme. Whereas in [33], the author proposed a deep reinforcement learning algorithm to maximize the weighted sum computation rate using a binary offloading scheme.

Implementation of Intelligent algorithms such as reinforcement and deep learning algorithms gain much attention from academia and industries in wireless communication [34] [35]. The core of these intelligent algorithms is data-driven rather than model-driven in conventional works. Deep learning has been used to boost the Maximum Probability Detector (MLD) detection effectiveness by leveraging the local correlation characteristics by the Deep Convolutional Neural Network (DCNN) [36]. In addition to deep learning, Some authors also considered the reinforcement learning algorithm to optimize the transmit power or tackle the attacker working in different modes like spoofing, interference, and eavesdropping [37]. Whereas the author in [38] uses Q-learning: a model-free reinforcement learning algorithm to suppress the attacker's attack, using the channel state information of attacker and antenna processing techniques.

\section{B. Motivation and Contribution}

A significant number of efforts have been made regarding the optimal allocation of resources and offloading schemes to enhance the system's performance using a mobile edge cloud. The main aim of MEC is to reduce the processing time of the task to meet the requirement of the resourcehungry application. In [24], the author states that the partial offloading scheme outperforms the binary offloading scheme using energy efficiency as a performance metric by assuming unlimited resources at MEC. So in this work, we considered the hybrid approach: partial offloading scheme, in which the task is divided into two portions, one portion of the task is computed locally at the devices using its computational resources and the other part is offloaded to MEC for further computations. To the best of our knowledge, it is the first attempt that studies the optimal task segmentation to minimize the computational task time using an algorithm that works on the statistical properties that avoid premature convergence using the rank-based selection method. The main contributions of this work are summarized as follows:

1) The proposed framework is to be devised as the optimal allocation of resources like local chip computational frequency, edge chip computational frequency, optimal task segmentation, and transmission power among devices to minimize the task's computational time.

2) An algorithm is designed for optimal allocation of the resources like local and edge computational capabilities, transmission power, and optimal task segmentation that optimize the function by keeping track of statistical properties of each candidate of the population.

3) A fundamental trade-off between the hybrid approach: partial offloading scheme and edge computing are analyzed. Results reveal that as the requirement increases, our proposed model performs more effectively than edge computing only.

4) The impact of the device's computational capability, volume of data, and computational cycle requirement on task segmentation is analyzed. Results demonstrate that optimal segmentation can effectively reduce the delay and increase the system's performance as compared to Edge computational scheme.

The rest of the paper is organized as follow: System Model is explained in section II whereas optimal resource allocation algorithm and results are discussed in Section III, and finally, the conclusion is drawn in section IV

\section{SYSTEM MODEL}

In this work, the MEC system is considered, where a $N$ number of users are distributed uniformly, as shown in Figure 1. These small devices consist of a single antenna connected to the MEC: provide services to low power devices like computational capabilities, connectivity, and storage. Besides, these devices have limited computation capabilities. In contrast, these devices collect a large volume of data from practical scenarios such as surveillance and security monitoring system, traffic control, lighting, pollution control, health, food, and water quality management. To meet the Quality of Experience (QoE), MEC located in their vicinity allow devices to offload their extensive tasks. This work aims to minimize the computation time of the task by using a partial offloading scheme. We define $\lambda_{n}$ the portion of the task computed locally for ease of simplicity, while $\pi_{n}=\left(1-\lambda_{n}\right)$ represents the remaining portion of the task. Rests of the parameters are explained below.

\section{A. Communication Model}

In the communication model, we consider the up-link data rate $r_{n}$ at which users offload their task to MEC. The channel $h_{n}$ between the devices and MEC is block faded, means it remains constant for an entire period of $T$. Mathematically, the data rate represented as:

$$
r_{n}=B \log _{2}\left(1+\frac{p_{n} h_{n}}{\sigma^{2}}\right)
$$




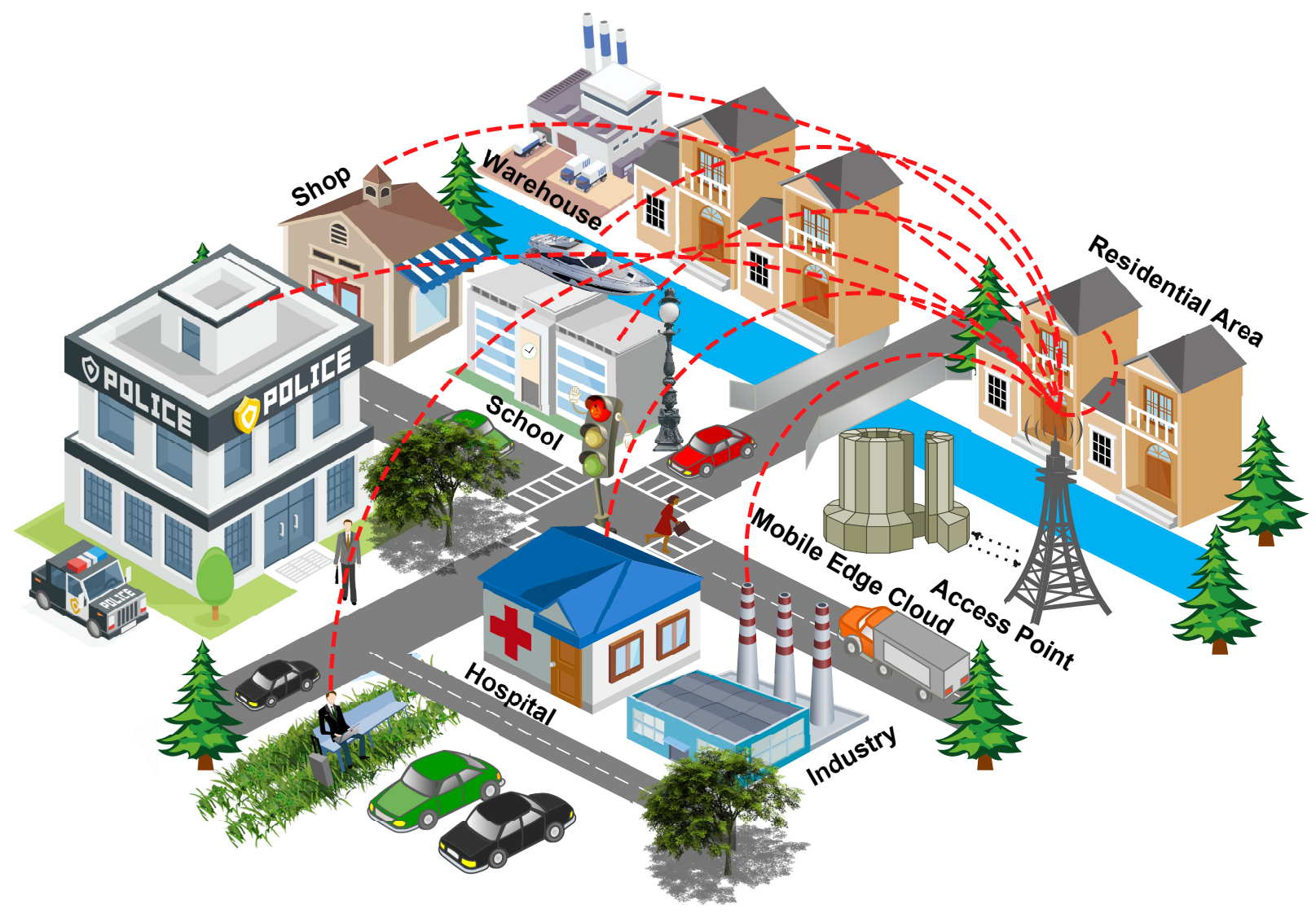

Fig. 1: System Model of Mobile Edge Cloud system

In Equation (1), $p_{n}$ and $B$ represent the transmitted power and channel bandwidth, respectively, whereas $\sigma^{2}$ represents the noise power of devices. Time consumed while offloading the task to MEC represented as follow,

$$
t_{n}^{T}=\frac{\pi_{n} s_{n}}{r_{n}}
$$

In Equation 2, $s_{n}$ represents the size of raw data collected by devices. Further, energy consumed while placing the task at MEC is as follow:

$$
e_{n}^{T}=\frac{p_{n} \pi_{n} s_{n}}{r_{n}}
$$

\section{B. Task Placement Model}

In order to meet the QoS or QoE, the task is either handled locally using local computational resources or at the MEC. To compute the task at the MEC it is needed to offload using partial offloading scheme or binary offloading scheme. In this work, we focus on a partial offloading scheme.

\section{Local Computation}

Local computation represents the scenario where the task is completed locally at the device using finite computational capabilities $f_{\max }^{L}$. Whereas $t_{n}^{L}$ represents the processing time of local computation.

$$
t_{n}^{L}=\frac{\lambda_{n} w_{n}}{k_{n}^{L} f_{\max ^{L}}}
$$

In Equation (4), $w_{n}$ represents the number of cycles required to compute the task. At the same time, $k_{n}^{L}$ represent the portion of local resources allocated to device to compute the task.

$$
e_{n}^{L}=\rho_{n} \lambda_{n} w_{n}
$$

Equation (5) represents the energy consume while computing the task locally whereas $\rho_{n}$ represents the constant circuit energy consume per CPU cycle.

\section{Edge Computing}

Edge computing emerges as an effective solution to overcome the hurdles face by devices. Edge computing provides computational capabilities on-demand fashion and allows devices to offload their computational task. Time consumes to compute the MEC task comprises processing time and offloading time can be obtained as follows.

$$
t_{n}^{E}=\frac{\pi_{n} w_{n}}{k_{n}^{E} f_{\max }^{c}}+\frac{\pi_{n} s_{n}}{r_{n}}
$$

In Equation (6), $f_{\max }^{c}$ represents the maximum computational capabilities of MEC. Whereas $k_{n}^{E}$ representing the portion of resources allocated to MEC's task place for computation purposes.

\section{E. Problem Formulation}

In this paper, we consider the partial offloading scheme to place tasks to MEC for computation while considering MEC's 
computational capabilities and finite battery capacity of devices. Our objective is to minimize tasks' duration by optimal allocation of resources among devices and MEC. The corresponding variables of computational resource allocation for both devices and MEC is as follow: $K^{E}=\left\{k_{1}^{E}, k_{2}^{E}, k_{3}^{E} \ldots k_{n}^{E}\right\}$ and $K^{L}=\left\{k_{1}^{L}, k_{2}^{L}, k_{3}^{L} \ldots k_{n}^{L}\right\}$ respectively. The optimal power allocation vector and portion of data computed locally is represented $P=\left\{p_{1}, p_{2}, p_{3} \ldots p_{n}\right\}$ and $\lambda=\left\{\lambda_{1}, \lambda_{2}, \lambda_{3} \ldots \lambda_{n}\right\}$ respectively. Thus, our objective is to minimize processing time by optimal allocation of resources among MEC and users. Mathematically we define our problem as follow:

$$
\begin{aligned}
& \text { P1: } \min _{\lambda, P, K^{L}, K^{E}} \sum_{n=1}^{N}\left(\frac{\pi_{n} w_{n}}{k_{n}^{E} f_{\max }^{c}}+\frac{\pi_{n} s_{n}}{r_{n}}+\frac{\lambda_{n} w_{n}}{k_{n}^{L} f_{\max }^{l}}\right) \\
& \text { subject to: } \\
& C_{1}: \frac{p_{n} \pi_{n} s_{n}}{r_{n}}+\rho_{n} \lambda_{n} w_{n} \leq E_{M a x}, \forall n \\
& C_{2}: \sum_{n=1}^{N} k_{n}^{E} \leq 1 \\
& C_{3}: 0 \leq k_{n}^{L} \leq 1, \forall n \\
& C_{4}: 0 \leq \lambda_{n} \leq 1, \forall n \\
& C_{5}: p_{n}>0, k_{n}^{E}>0, \forall n
\end{aligned}
$$

In Equation (7), our objective is to minimize the total duration to compute the specific task by optimally allocating resources like transmission power, local computational resources, edge computational resources, and task segmentation. While ensuring the set of constraints needs to be satisfied. $C_{1}$ states that energy consumed while computing tasks locally and the energy consumed while offloading the task should be less than the maximum battery capacity. $C_{2}$ states the sum of resources allocated to the task place at the edge should be less or equal to $1 . C_{3}$ states that the lower and upper bound of a portion of resources allocated to the task is computed locally. $C_{4}$ represents the lower and upper bound of the task segmentation variable. $C_{5}$ represents the lower bound of transmission power and computational resources allocated to the task at MEC.

\section{F. Transformation of Problem}

As we have seen in the objective function in (7), it contains $4 N$ decision variables and non-convex in nature. That makes it hard to find the closed-form solution of the Problem 1. To determine the variables, we need to find the optimal values of $\lambda$ by fixing the rest of the parameters. Task segmentation mainly depends on the local chip computational capability, the volume of data, and the number of cycles needed to compute data volume. Based on this, we derive an optimal task segmentation strategy as given below:

$$
\lambda_{n}=\frac{k_{n}^{L} f_{\max }^{L}\left(r_{n} w_{n}+k_{n}^{E} f_{\max }^{E} s_{n}\right)}{w_{n} k_{n}^{E} f_{\max }^{E} r_{n}+w_{n} k_{n}^{L} f_{\max }^{L} r_{n}}
$$

Proof: Please refer to APPENDIX A.

Equation (8) reveals that the task segmentation on devices mainly depends on the computational resources allocated to the task, the volume of data sense by the devices', and the sensed task's computational requirement as explained below:
1) Impact of local computational resources on task segmentation: The local computational resource defines as the number of computational resources allocated by the device to compute the task is represented by $k_{n}^{L} f_{\max }^{L}$. If the devices constitute computational resources that's not enough to handle the task locally, then devices offload the portion or complete the task to MEC located in their vicinity. Mathematically it can be expressed as follow:

$$
\lim _{k_{n}^{L} f_{\max }^{L} \rightarrow 0} \lambda_{n}=0
$$

On the other hand:

$$
\lim _{k_{n}^{L} f_{\max }^{L} \rightarrow \infty} \lambda_{n}=1
$$

Figure 2a, shows that, for low computational capabilities devices, the value of $\lambda_{n}$ is zero, mean whole the task is offloaded to MEC for computational purpose because the devices computational capability is not enough to handle the task locally and introduce latency in the system. Besides, as the device's computational resource increases, devices start segmentation the task at the end; when devices have enough computational resources, they begin computing the task as a whole locally.

2) Impact of computational cycles requirement on task segmentation: The number of cycles requirement, defined as the number of cycles required to compute a specific task, is represented by $w_{n}$. It is an important parameter that has an enormous impact on task segmentation. As we know, the decision to offload the task is taken by the devices themselves. Devices have enough computation capability that they can handle the small number of cycles. In contrast, as the number of cycle requirements increases, devices are not able to handle or they have not enough computation to perform it in minimal time, so they start offloading it to MEC. Mathematically it can be expressed as follow:

$$
\lim _{w_{n} \rightarrow 0} \lambda_{n}=1
$$

Whereas large number cycles requirement leads to offloading the complete task to MEC located in their vicinity as shown below:

$$
\lim _{w_{n} \rightarrow \infty} \lambda_{n}=0
$$

Figure $2 \mathrm{~b}$ shows the comparative analysis of task segmentation and the number of cycle requirements. It is seen that as for a small number of cycle requirements, the value of $\lambda_{n}=1$ means the whole task is computed locally. On the other hand, as the number of cycles requirement increases, devices' computational capability is insufficient to handle the task. They start offloading; thus, the value of $\lambda_{n}=0$ for massive number of cycles requirement.

3) Impact of volume of data on task segmentation: The volume of data $s_{n}$ defines as the number of bits sensed by the devices that need to be computed in minimal time. It shows the tremendous effect on task segmentation as shown below:

For small number of bits:

$$
\lim _{s_{n} \rightarrow 0} \lambda_{n}=1
$$


For large volume of data:

$$
\lim _{s_{n} \rightarrow \infty} \lambda_{n}=0
$$

Equations (13) and (14) reveals that for a small number of sensed bits, the value of $\lambda_{n}=0$ means the task as a whole is offloaded to MEC because energy consumes to offload a small number of bits is also small. On the other hand, as the number of sensed bits increases, the energy consumes while offloading numerous bits also increases. To validate the constraint $C_{1}$ mention in (7), devices start to compute it locally as a results value of $\lambda_{n}=0$. This tremendous effect can be seen in Figure $2 \mathrm{c}$ : shows the comparative analysis between the volume of data and task segmentation.

Thus, integrating the optimal value of $\lambda_{n}$ calculated in (8) with (7) leads toward a convex non-linear problem equivalent to Problem 1 as given below.

$$
\text { P2: } \min _{P, K^{L}, K^{E}} \sum_{n=1}^{N}\left(\frac{\pi_{n} w_{n}}{k_{n}^{E} f_{\max }^{c}}+\frac{\pi_{n} s_{n}}{r_{n}}+\frac{\lambda_{n} w_{n}}{k_{n}^{L} f_{\max }^{l}}\right)
$$

subject to:

$$
C_{1}: C_{3} \text { and, } C_{5}
$$

Thus the Objective function mentioned in (15) is convex, and the optimization problem is a convex non-linear optimization problem.

Proof: Please refer to APPENDIX B.

\section{RESUlts AND Discussion}

\section{A. Optimal Resource Allocation}

The problem regarding optimal allocation of resources formulated in (15) is nonlinear and convex, in order to minimize the task duration, we define an algorithm 1 named Estimation of Optimal Resource Allocator (EORA) with the complexity of $O\left(n^{2}\right)$. EORA optimizes the function by using the statistics of each candidate from the population $\Xi$. After calculating the statistical parameter, it does not need to hold the population for the next generation. The next generation's population is generated based upon the fittest candidates of the current generation population, and it is repeatedly iterated continuously unless termination criteria is to be met. So EORA is a population-based algorithm that discards at least part of each generation's population and replaces it using highly-fit individuals' statistical properties.

As seen in algorithm 1, it comprises of several steps. Ere proceeding toward execution of the algorithm, first step is to initialize known parameters. After that, compute each candidate's fitness and select $S_{\Xi}$ candidate from $\Xi$ using the rank base selection (RBS): also known as rank weighting, then the individual is sorted based upon its ranking rather than fitness values. The rank-based selection method ranks the individual based upon its fitness by giving the rank $\Xi_{\text {size }}$ to the best candidate and 1 to the worst candidate. In contrast, the average candidate is ranked with $\frac{\Xi_{s i z e}+1}{2}$, and then the selection is performed based upon its rank. Base upon the rank matrix, we calculate the probability of each candidate's

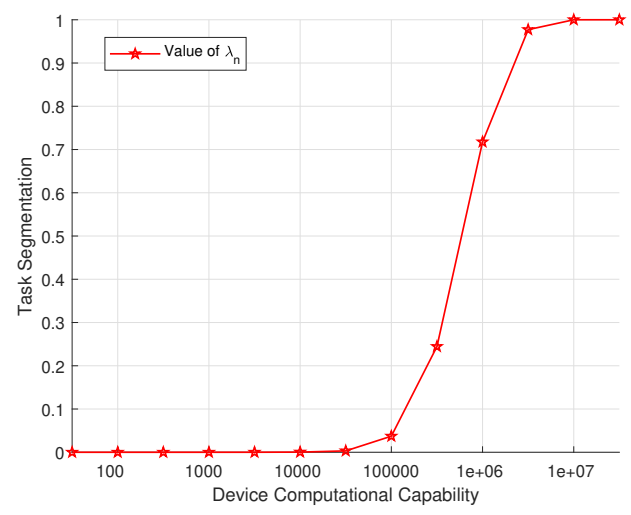

(a) Device computational capability

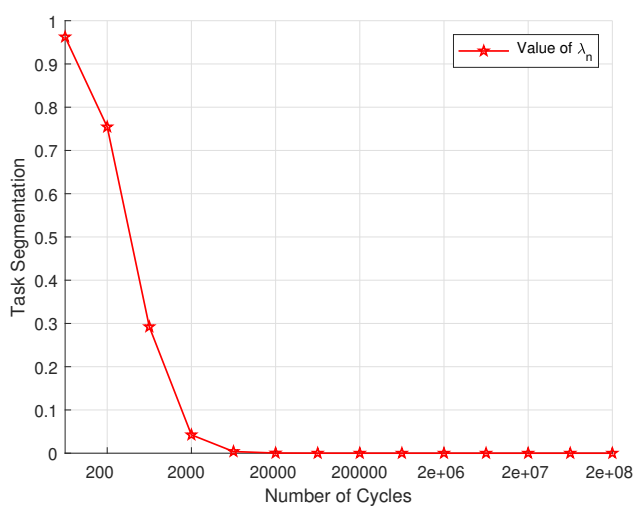

(b) Number of cycles

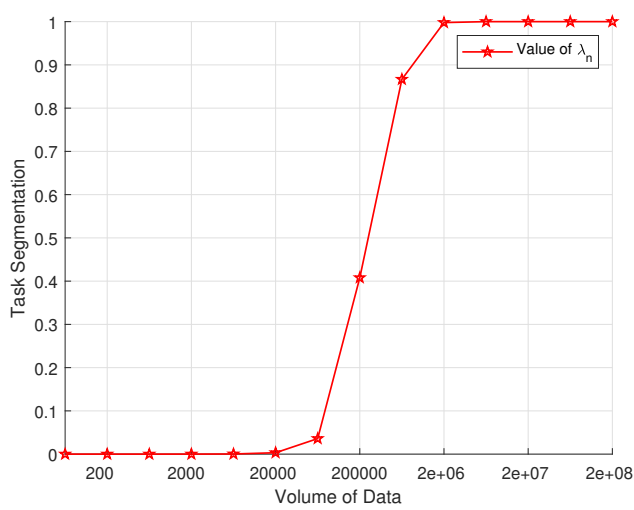

(c) Volume of Data

Fig. 2: Illustration of the optimal task segmentation strategy capability.

section having a sum equal to 1 by using the equation as given below.

$$
\operatorname{Pr}\left(\alpha_{i}\right)=\theta+\eta R\left(\alpha_{i}\right), \quad \forall \Xi
$$

In Equation (16), $\theta$ and $\eta$ are the user defined parameters. Whereas the $R$ represents the rank matrix of candidate $\alpha_{i} \in P$. By assuming that all the probabilities are non-negative, we calculate the selection pressure as follows.

$$
\chi=\frac{\operatorname{Pr}_{\text {best }}}{\operatorname{Pr}_{\text {average }}}
$$




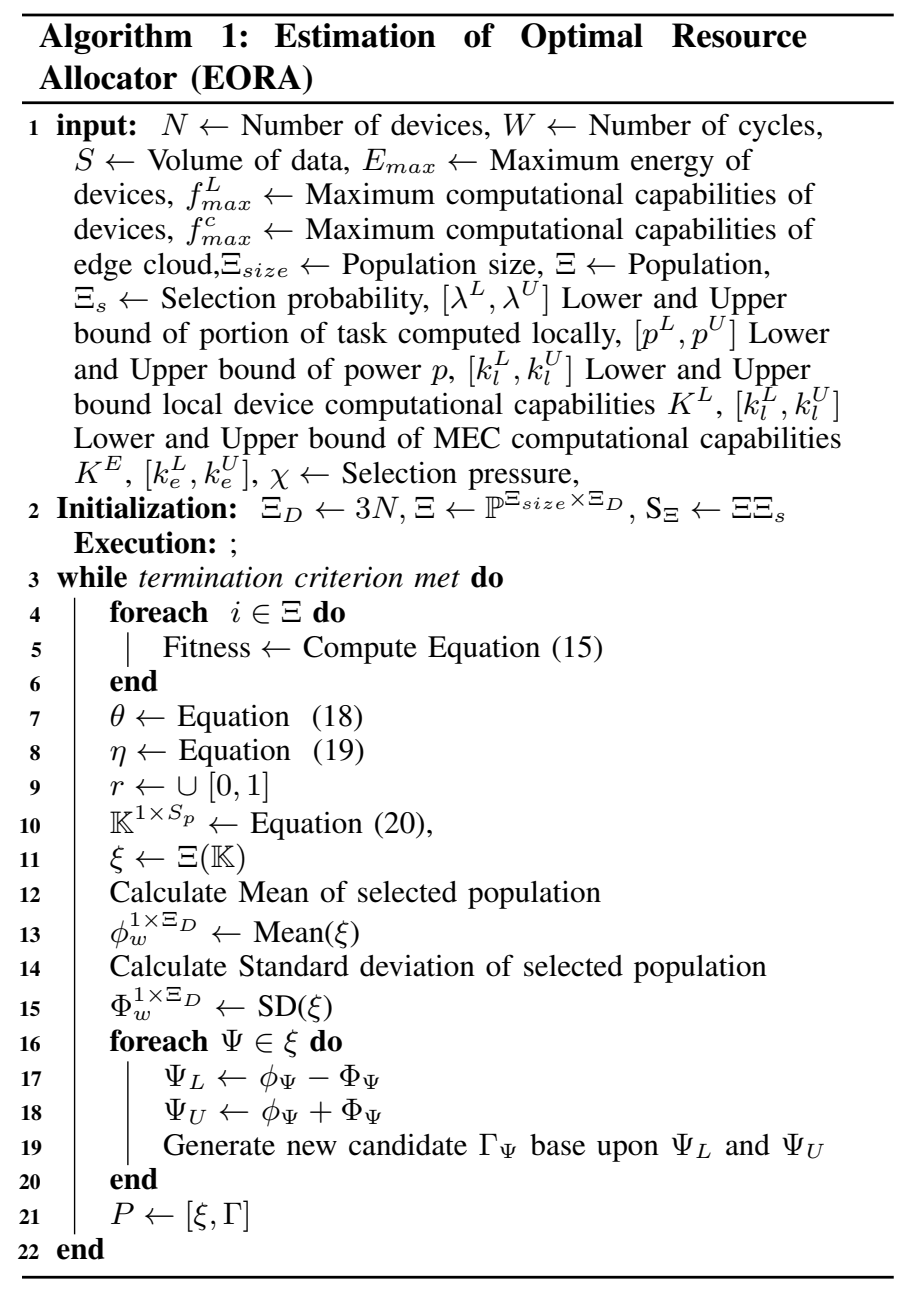

Selection pressure $\chi$ is a vital performance metric that quantifies the relative probability upon which a highly-fit individual will participate in recombination. To avoid premature convergence, we set the selection pressure low initially, increasing over time. The value of $\chi$ can be customized by different values of $\theta$ and $\eta$ using the equation (18) and (19) respectively given below.

$$
\begin{gathered}
\theta=\frac{2 \Xi_{\text {size }}-\chi\left(\Xi_{\text {size }}+1\right)}{\Xi_{\text {size }}\left(\Xi_{\text {size }}+1\right)} \\
\eta=\frac{2(\chi-1)}{\Xi_{\text {size }}\left(\Xi_{\text {size }}-1\right)}
\end{gathered}
$$

To calculate the index of selected population. Generate a random number $r \in \cup[0,1]$. For simplicity we say that $k=\Xi_{\text {size }}$, total number of candidate in the population $P$.

$$
k=\frac{-2 \theta-\eta+\sqrt{(2 \theta+\eta)^{2}+8 \eta r}}{2 \eta}
$$

$k$ represent the index of selected candidate.

Proof: Please refer to APPENDIX C.

After selecting the best candidates as stored in $\xi$, the next step is to calculate the mean and standard deviation of the selected population and store the results in $\phi$ and $\Phi$, respectively. Using the mean and variance, update each candidate's
TABLE I: Simulation Parameters.

\begin{tabular}{|c|c|l|}
\hline Parameter Name & Symbol & Value \\
\hline Bandwidth & $B$ & $2 \mathrm{Mhz}$ \\
\hline Number of devices & $N$ & 200 \\
\hline Number of cycles & $w_{n}$ & {$[0.2,2]$ Mcycles } \\
\hline Volume of data & $s_{n}$ & $20,200]$ Mbits \\
\hline Device computational capability & $f_{\max }^{l}$ & $1 \mathrm{Mcycles} / \mathrm{sec}$ \\
\hline Edge computational capability & $f_{\max }^{c}$ & $0.25 \mathrm{Tcy}$ cles $/ \mathrm{sec}$ \\
\hline Maximum Energy of devices & $E_{\max }$ & $2 \mathrm{joules}$ \\
\hline Static circuit power & $\rho_{n}$ & $900 \mu \mathrm{W}$ \\
\hline
\end{tabular}

lower and upper bound and, based on this, generates a new population for the next generation. This process continues until the termination criteria is to be met.

\section{B. Discussion}

This section presents the numerical results to validate the proposed scheme. Extensive Montecarlo simulations were carried out by EORA using $M A T L A B$. Furthermore, to validate the proposed algorithm results, results are compared with the algorithm name Mesh Adaptive Direct Search algorithm (MADs) [39]. We consider the $N$ number of devices distribute randomly and one MEC located at the AP. These devices are connected to MEC through wireless channel $h$. The channel between devices and MEC is considered as block-fading, which means it remains constant for the entire period. The total bandwidth of the network is $B=2 \mathrm{MHz}$. Thus for each device's, the volume of tasks and cycles is needed to compute following a uniform distribution with $s_{n} \in[20,200]$ Mbits and $w_{n} \in[0.2,2]$ Mcycles. Whereas the maximum computational capabilities of edge and devices are $f_{\max }^{c}=0.25 \mathrm{THz}$ and $f_{\max }^{l}=1 \mathrm{MHz}$, respectively. Other essential simulations parameters are listed in Table I.

Figure 3 represents the hybrid approach's comparative analysis: partial offloading scheme and edge computation only by using computational time as a performance metric. Results demonstrate that for a small number of users, the performance of the hybrid approach and edge computational scheme is the same. In contrast, as the number of users increases, the hybrid approach outperforms the edge computational scheme. As mentioned in (15), the edge divides the computational capabilities among the number of users connected. Simultaneously a portion of computational resources allocated to each user's task at the edge is enormous than its computational capabilities, as shown in Figure 3a. Whereas successive offloading to the edge introduces overhead at the MEC and thus introduces latency in the system as the number of users in the system increases.

On the other hand, as the volume of the task and computational cycle requirement increases, the hybrid approach starts performing better even for a small number of users, as shown in Figure 3b. This behavior is because offloading a large volume of data as a whole to MEC required a large amount of energy, and it also takes much time. In the hybrid approach, tasks are divided into two portions; one portion of the task is computed locally, and the other part is offloaded to MEC for computational purposes. As a result, it takes less time than the edge computational scheme. These results reflect the fundamental trade-off between offloading only and the 


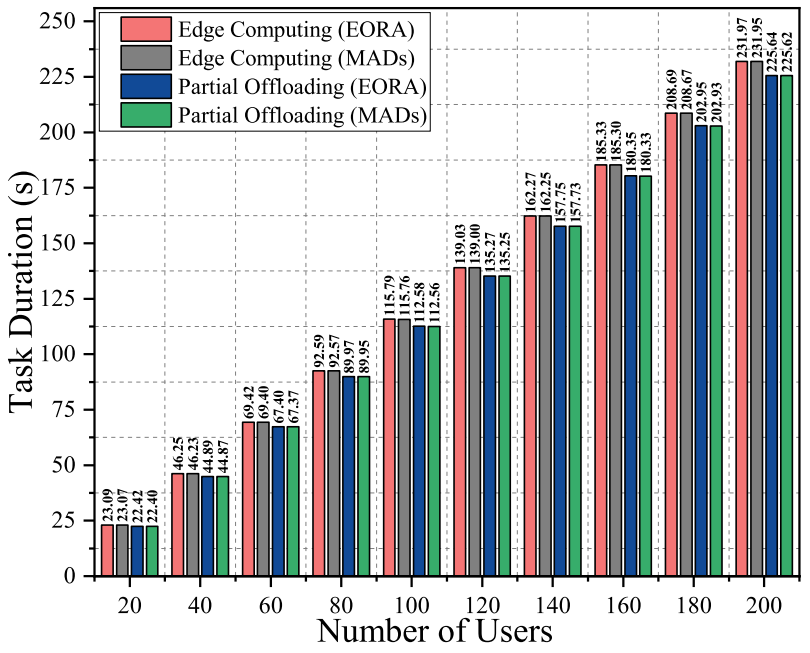

(a) $w_{n}=1.2$ Mcycles, $s_{n}=60$ Mbits

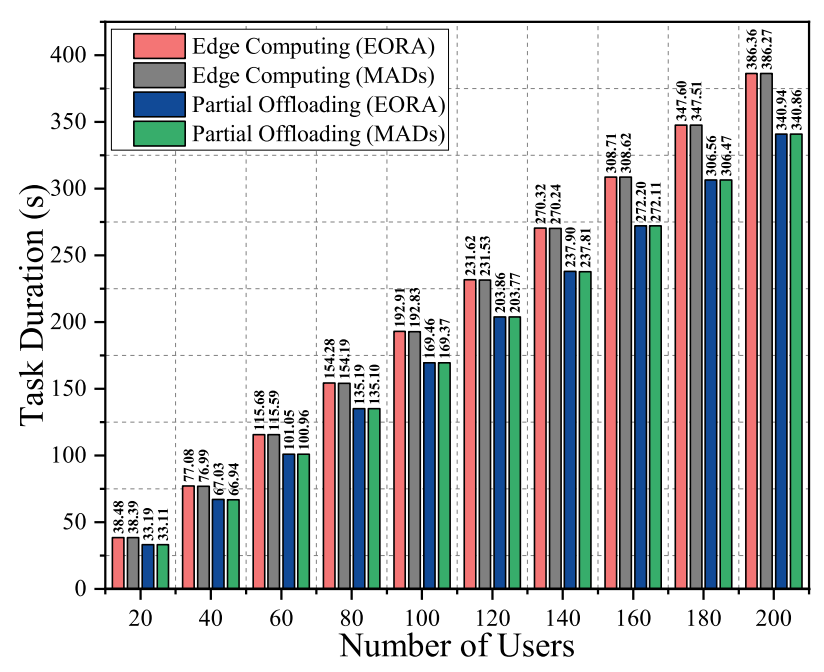

(b) $w_{n}=1.6$ Mcycles, $s_{n}=100$ Mbits

Fig. 3: Comparison of task duration and number of users using hybrid and edge computational model.

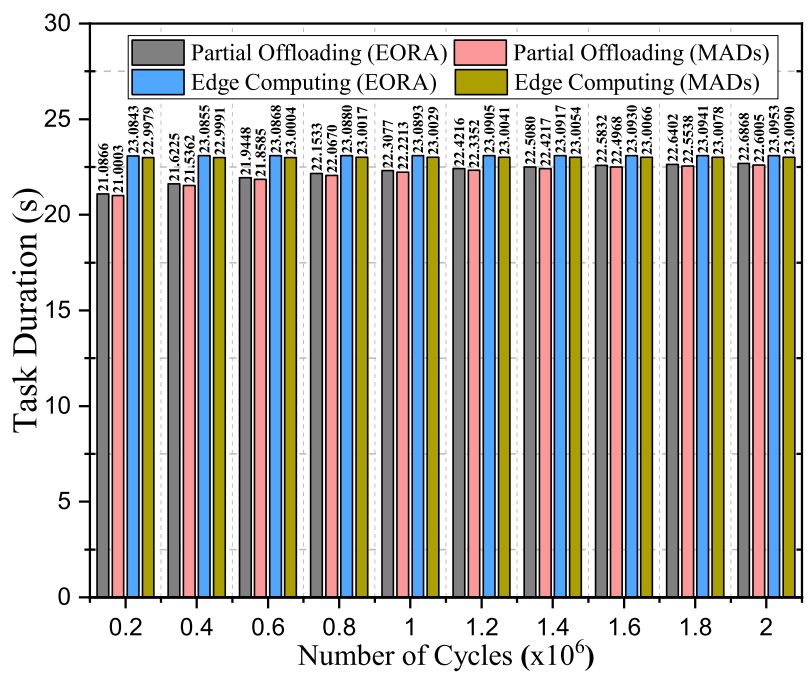

(a) $n=20$ Users, $s_{n}=60$ Mbits

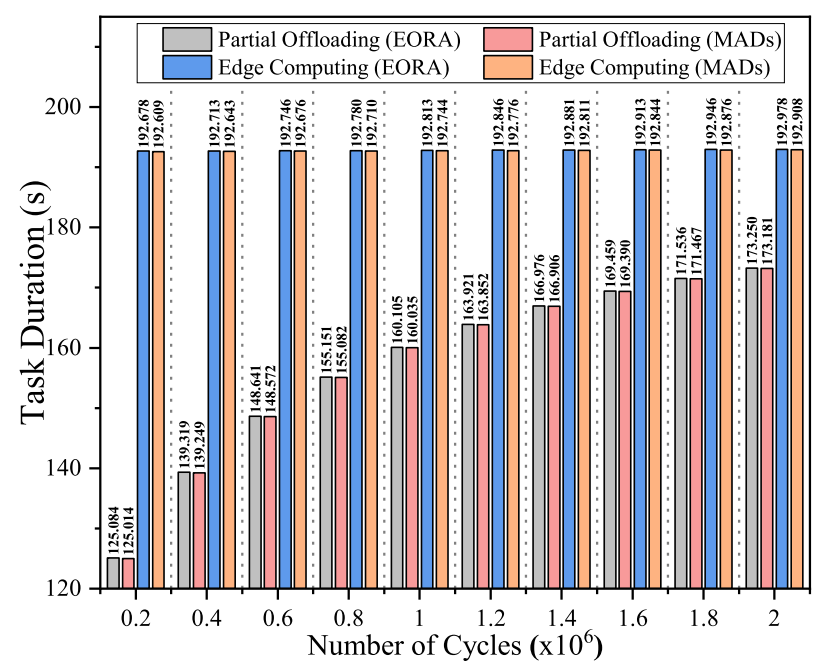

(b) $n=100$ Users, $s_{n}=100$ Mbits

Fig. 4: Comparison of task duration and number of cycles using hybrid and edge computational model.

hybrid approach. For a small number of users, low volume of data, low computational cycle requirement, Edge computational scheme's performance, and a hybrid approach are the same. On the other hand, as the number of users, volume of the task, and computational cycles requirement increases, the hybrid approach starts performing better. It reflects that the partial offloading model becomes more evident with the growing number of devices, volume, and computational cycles requirement. Simultaneously, It reduces the delay and increase the QoE of devices.

Figure 4 represents the hybrid approach's comparative analysis: partial offloading scheme and edge computation using computational time as a performance metric across the number of cycle requirements. Results demonstrate that by varying the number of cycles, the task's computational time increased because large cycles require extensive computation to complete the task. Simultaneously, outcomes illustrate that the hybrid approach outperforms the edge computational scheme. This tremendous effect is shown in Figure 4a. Results reveal that successive offloading in edge computation scheme leads toward an increase in the system's overhead and thus introduce latency in the system, which is a critical parameter of a $5^{\text {th }}$ generation communication system. On the other hand, the hybrid scheme's performance approaches to the edge computational scheme as the computational cycle requirement increases. In contrast, as the number of users and volume of tasks increases, the difference between both schemes' performance can be more promptly in Figure 4a. The reason for the trend is that offloading a large number of users task increase overhead on the system, simultaneously offloading a large volume of data to MEC which also requires more time and utilizes extensive energy. In comparison, the hybrid approach outperforms the edge computation as the number of cycles increases because offloading the massive number 


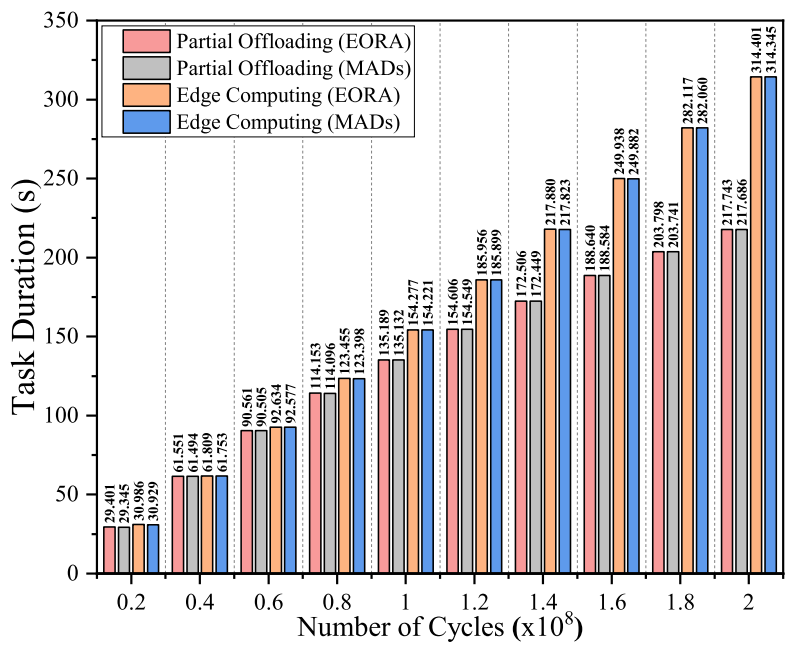

Fig. 5: Comparison of task duration and volume of data using hybrid and edge computation model by setting, Cycles $=1.6$ Mcycles and Users $=40$

of cycles requires considerable time and energy. The hybrid approach emerges as an effective solution to meet the massive requirement of cycles. Segmentation of tasks is done optimally into two parts; both are computed parallel at the edge and the devices locally. Thus parallel computation of task results in completion on time within a short duration compared to edge computation.

This hybrid approach can be utilized more effectively in smart cities where the devices collect a large volume of delaysensitive data in real-time. A smart city's main aim is the optimal utilization of assets to provide the QoS to users that are always connected, fully aware, and auto-managed. To meet the QoS requirement, large volumes of raw data generated by these devices should be computed in minimal time. A portion of an extensive task offloaded to MEC lead to the minimization of computational task duration, as shown in Figure 3 and Figure 4. Results illustrate that as the task's volume increases, the proposed scheme outperforms the edge computation and reveals the fundamental trade-off between edge computation and the hybrid approach.

A comparative analysis of the edge computing and hybrid approach is done by varying the data volume to validate the trade-off. By analyzing the results, it is perceived that the performance of edge computation and a hybrid approach has the same for the minute volume of task, as shown in Figure 5. In contrast, as the number of sensed bits increases, the hybrid approach outperforms the edge computational scheme. This reason for the trend that because offloading a small number of bits consumes less energy and require less time, so it is better to compute it at the MEC. In contrast, as the number of computation bits increases, offloading in the edge computation scheme leads toward an increase in the computational time, whereas in the hybrid approach, it starts dividing the task into two portions. One portion of the task is computed locally at the devices while offloading the other part to MEC for further computation, as shown in Figure 5. This trend validates the fundamental trade-off between edge computation only and the hybrid approach.

\section{CONCLUSION}

In this work, a convex optimization problem is formulated to minimize the task's computational time by optimally allocating resources among local devices and mobile edge cloud. Two models name edge computation and hybrid approach: partial offloading scheme is considered. Besides this, it also reveals the fundamental trade-off between edge computation and a hybrid approach. Simulation results reveal that our proposed model is more efficient as compared to edge computation. Furthermore, we analyze the impact of task segmentation on systems' performance and find that optimal segmentation can effectively reduce the tasks' computational time compared to fixed or random segmentation.

\section{APPENDIX A}

\section{PROOF OF LEMMA 1}

We can prove the expression of $\lambda_{n}$ mention in (8) by analyzing the monotonically between task segmentation $\lambda_{n}$ and computational delay. In our problem, computational delay constitutes local computational time and edge computational time. As in (4), time consumed while computing the task locally at the devices is represented as $t_{n}^{L}=\frac{\lambda_{n} w_{n}}{k_{n}^{L} f_{\max L}}$. Time consumed while computing the task locally mainly depends on the value of $\lambda_{n}$, and locally computational time increases as the value of $\lambda_{n}$ increases. Whereas $\lambda_{n} \in[0,1]$, base upon this we can simply expressed local computational time as $t_{n}^{L} \in\left[0, \frac{w_{n}}{k_{n}^{L} f_{\max } L}\right]$.

Simultaneously, on the other hand edge computational time $t_{n}^{E}=\left(1-\lambda_{n}\right)\left(\frac{w_{n}}{k_{n}^{E} f_{\max }^{E}}+\frac{s_{n}}{r_{n}}\right)$ as mentioned in (6), also the function of $\lambda_{n}$. Whereas edge computational time shows the inverse relation with task segmentation $\lambda_{n}$ value. As the value of $\lambda_{n}$ increases, edge computational time decreases. Therefore, base upon $\lambda_{n} \in[0,1]$, we can express the value of edge computation time as $t_{n}^{E} \in\left[0, \frac{w_{n}}{k_{n}^{E} f_{\max }^{E}}+\frac{s_{n}}{r_{n}}\right]$. Due to the inverse relationship between local computation time and edge computation time, the minimum computation delay is archived only when:

$$
t_{n}^{L}=t_{n}^{E} .
$$

By considering the expression of local computational time and edge computation time:

$$
\begin{gathered}
\frac{\lambda_{n} w_{n}}{k_{n}^{L} f_{\max }^{L}}=\left(1-\lambda_{n}\right)\left(\frac{w_{n}}{k_{n}^{E} f_{\max }^{E}}+\frac{s_{n}}{r_{n}}\right) \\
\frac{\lambda_{n} w_{n}}{k_{n}^{L} f_{\max }^{L}}=\left(\frac{w_{n}}{k_{n}^{E} f_{\max }^{E}}+\frac{s_{n}}{r_{n}}\right)-\lambda_{n}\left(\frac{w_{n}}{k_{n}^{E} f_{\max }^{E}}+\frac{s_{n}}{r_{n}}\right) \\
\frac{\lambda_{n} w_{n}}{k_{n}^{L} f_{\max }^{L}}+\lambda_{n}\left(\frac{w_{n}}{k_{n}^{E} f_{\max }^{E}}+\frac{s_{n}}{r_{n}}\right)=\left(\frac{w_{n}}{k_{n}^{E} f_{\max }^{E}}+\frac{s_{n}}{r_{n}}\right)
\end{gathered}
$$

After some mathematical calculation, optimal task segmentation ratio expressed as follow:

$$
\lambda_{n}=\frac{k_{n}^{L} f_{\max }^{L}\left(r_{n} w_{n}+k_{n}^{E} f_{\max }^{E} s_{n}\right)}{w_{n} k_{n}^{E} f_{\max }^{E} r_{n}+w_{n} k_{n}^{L} f_{\max }^{L} r_{n}}
$$

This end the proof. 


\section{APPENDIX B \\ PROOF OF LEMMA 2}

To prove the Lemma 2, we need to verify the objective function mention in (15) is convex in nature by using the concept of the Hessian matrix. If the Hessian matrix produces a positive definite then (15) is convex in nature.

Therefore Hessian of $f(x)$ can be characterized as follow,

$$
\begin{gathered}
\nabla f=\left[\begin{array}{lll}
\frac{\partial f(x)}{\partial P} & \frac{\partial f(x)}{\partial K^{L}} & \frac{\partial f(x)}{\partial K^{E}}
\end{array}\right]^{t} \\
\nabla^{2} f\left[\begin{array}{ccc}
\frac{\partial^{2} f(z)}{\partial P^{2}} & \frac{\partial^{2} f(z)}{\partial P^{2} \partial K^{L}} & \frac{\partial^{2} f(z)}{\partial P^{2} \partial K^{E}} \\
\frac{\partial^{2} f(z)}{\partial K^{L} \partial P} & \frac{\partial^{2} f(z)}{\partial K^{L}} & \frac{\partial^{2} f(z)}{\partial K^{L} \partial K^{E}} \\
\frac{\partial^{2} f(z)}{\partial K^{E} \partial P} & \frac{\partial^{2} f(z)}{\partial K^{E} \partial K^{L}} & \frac{\partial^{2} f(z)}{\partial K^{E^{2}}}
\end{array}\right]
\end{gathered}
$$

After applying Linear algebra techniques, we find that all the entities are zero except diagonal elements as follows:

$$
\nabla^{2} f=\left[\begin{array}{ccc}
\frac{\partial^{2} f(x)}{\partial P^{2}} & 0 & 0 \\
0 & \frac{\partial^{2} f(x)}{\partial K^{L^{2}}} & 0 \\
0 & 0 & \frac{\partial^{2} f(x)}{\partial K^{E^{2}}}
\end{array}\right]
$$

Whereas the diagonal elements as given below,

$$
\begin{gathered}
\frac{\partial^{2} f(x)}{\partial P^{2}}=\frac{s_{n} h}{B^{2}\left(\sigma^{2}+P\right)}\left(\left(B \log _{2}\left(1+\frac{P h}{\sigma^{2}}\right)\right)^{-3}+1\right)>0 \\
\frac{\partial^{2} f(x)}{\partial K^{L^{2}}}=\frac{2 w_{n}}{K^{L^{3} f_{\max }^{L}}>0} \\
\frac{\partial^{2} f(x)}{\partial K^{E^{2}}}=\frac{2 w_{n}}{K^{E^{3} f_{\max }^{E}}}>0
\end{gathered}
$$

Equation (29) to (31) reflects that all the diagonal elements are positive, whereas all the other aspects of the Hessian matrix are zeros. Thus, the determinant of the Hessian matrix is also greater than zero $\left|\nabla^{2} f\right|>0$, this reveals that the objective function mention in (15) is convex.

This end the proof.

\section{APPENDIX C \\ PROOF OF LEMMA 3}

The purpose is to avoid looping and to find the index of candidate. For this a random number $r \cup[0,1]$ is generated, such that the sum of probabilistic function of all the candidate is approximately equal to $r$ as shown in (32).

$$
\sum_{i=1}^{k} \operatorname{Pr}\left(\alpha_{i}\right) \approx r
$$

Put (16) in (32).

$$
\sum_{i=1}^{k}\left(\theta+\eta R\left(\alpha_{i}\right)\right) \approx r
$$

Normalizing the selection probabilities so that they sum equal to $r$.

$$
\begin{gathered}
\theta k+\frac{\eta(k+1)}{2} \approx r \\
2 \theta+\eta k^{2}+\eta k-2 r \approx 0
\end{gathered}
$$

After some mathematical calculation, (35) become the simple quadratic equation and can be solved by simple completing square method.

$$
\begin{gathered}
k^{2}+\left(\frac{2 \theta+\eta}{\eta}\right) k-\frac{2 r}{\eta} \approx 0 \\
k^{2}+\left(\frac{2 \theta+\eta}{\eta}\right) k+\left(\frac{2 \theta+\eta}{2 \eta}\right)^{2}-\frac{2 r}{\eta}-\left(\frac{2 \theta+\eta}{2 \eta}\right)^{2} \approx 0 \\
\left(k+\frac{2 \theta+\eta}{2 \eta}\right)^{2} \approx \frac{8 \eta r+(2 \theta+\eta)^{2}}{4 \eta^{2}} \\
\pm\left(k+\frac{2 \theta+\eta}{2 \eta}\right) \approx \frac{\sqrt{8 \eta r+(2 \theta+\eta)^{2}}}{2 \eta}
\end{gathered}
$$

Neglect the negative values because $k$ is the index.

$$
k=\frac{\sqrt{8 \eta r+(2 \theta+\eta)^{2}}-(2 \theta+\eta)}{2 \eta}
$$

This end the proof.

\section{REFERENCES}

[1] W. U. Khan, F. Jameel, N. Kumar, R. Jäntti, and M. Guizani, "Backscatter-enabled efficient V2X communication with non-orthogonal multiple access," IEEE Transactions on Vehicular Technology, vol. 70, no. 2, pp. 1724-1735, 2021.

[2] Z. Zhou, X. Chen, E. Li, L. Zeng, K. Luo, and J. Zhang, "Edge intelligence: Paving the last mile of artificial intelligence with edge computing," Proceedings of the IEEE, vol. 107, no. 8, pp. 1738-1762, 2019.

[3] A. Hegyi, H. Flinck, I. Ketyko, P. Kuure, C. Nemes, and L. Pinter, "Application orchestration in mobile edge cloud: placing of iot applications to the edge," in 2016 IEEE 1st International Workshops on Foundations and Applications of Self* Systems (FAS*W). IEEE, 2016, pp. 230-235.

[4] A. Mahmood, M. Q. Usman, K. Shahzad, and N. Saddique, "Evolution of optimal 3d placement of uav with minimum transmit power," vol, vol. 7, pp. 13-18, 2019.

[5] J. Ren, D. Zhang, S. He, Y. Zhang, and T. Li, "A survey on endedge-cloud orchestrated network computing paradigms: Transparent computing, mobile edge computing, fog computing, and cloudlet," $A C M$ Computing Surveys (CSUR), vol. 52, no. 6, pp. 1-36, 2019.

[6] W. U. Khan, J. Liu, F. Jameel, V. Sharma, R. Jäntti, and Z. Han, "Spectral efficiency optimization for next generation noma-enabled iot networks," IEEE Transactions on Vehicular Technology, vol. 69, no. 12, pp. 15284 15297,2020

[7] M. Li, P. Si, and Y. Zhang, "Delay-tolerant data traffic to softwaredefined vehicular networks with mobile edge computing in smart city," IEEE Transactions on Vehicular Technology, vol. 67, no. 10, pp. $9073-$ 9086, 2018.

[8] Y. Deng, Z. Chen, X. Yao, S. Hassan, and J. Wu, "Task scheduling for smart city applications based on multi-server mobile edge computing," IEEE Access, vol. 7, pp. 14410-14421, 2019.

[9] X. Huang, R. Yu, J. Kang, Y. He, and Y. Zhang, "Exploring mobile edge computing for $5 \mathrm{~g}$-enabled software defined vehicular networks," IEEE Wireless Communications, vol. 24, no. 6, pp. 55-63, 2017.

[10] A. Yousefpour, C. Fung, T. Nguyen, K. Kadiyala, F. Jalali, A. Niakanlahiji, J. Kong, and J. P. Jue, "All one needs to know about fog computing and related edge computing paradigms: A complete survey," Journal of Systems Architecture, vol. 98, pp. 289-330, 2019

[11] Y. Zhang, X. Lan, Y. Li, L. Cai, and J. Pan, "Efficient computation resource management in mobile edge-cloud computing," IEEE Internet of Things Journal, vol. 6, no. 2, pp. 3455-3466, 2018.

[12] A. Ceselli, M. Premoli, and S. Secci, "Mobile edge cloud network design optimization," IEEE/ACM Transactions on Networking, vol. 25, no. 3, pp. 1818-1831, 2017.

[13] D. Wang, B. Bai, K. Lei, W. Zhao, Y. Yang, and Z. Han, "Enhancing information security via physical layer approaches in heterogeneous iot with multiple access mobile edge computing in smart city," IEEE Access, vol. 7, pp. 54 508-54 521, 2019. 
[14] Y. Zhang, X. Lan, J. Ren, and L. Cai, "Efficient computing resource sharing for mobile edge-cloud computing networks," IEEE/ACM Transactions on Networking, 2020.

[15] X. Wang, Y. Han, C. Wang, Q. Zhao, X. Chen, and M. Chen, "In-edge ai: Intelligentizing mobile edge computing, caching and communication by federated learning," IEEE Network, vol. 33, no. 5, pp. 156-165, 2019.

[16] B. Nguyen, N. Choi, M. Thottan, and J. Van der Merwe, "Simeca: Sdnbased iot mobile edge cloud architecture," in 2017 IFIP/IEEE Symposium on Integrated Network and Service Management (IM). IEEE, 2017, pp. 503-509.

[17] W. Z. Khan, E. Ahmed, S. Hakak, I. Yaqoob, and A. Ahmed, "Edge computing: A survey," Future Generation Computer Systems, vol. 97, pp. 219-235, 2019.

[18] Z. Ning, P. Dong, X. Wang, X. Hu, L. Guo, B. Hu, Y. Guo, T. Qiu, and R. Kwok, "Mobile edge computing enabled 5g health monitoring for internet of medical things: A decentralized game theoretic approach," IEEE J. Sel. Areas Commun., pp. 1-16, 2020.

[19] Y. Jararweh, S. Otoum, and I. Al Ridhawi, "Trustworthy and sustainable smart city services at the edge," Sustainable Cities and Society, vol. 62, p. 102394, 2020.

[20] R. Zhao, X. Wang, J. Xia, and L. Fan, "Deep reinforcement learning based mobile edge computing for intelligent internet of things," Physical Communication, vol. 43, p. 101184, 2020.

[21] Z. Zhou, H. Yu, C. Xu, Z. Chang, S. Mumtaz, and J. Rodriguez, "Begin: Big data enabled energy-efficient vehicular edge computing," IEEE Communications Magazine, vol. 56, no. 12, pp. 82-89, 2018.

[22] Z. Guan, Y. Zhang, G. Si, Z. Zhou, J. Wu, S. Mumtaz, and J. Rodriguez, "Ecosecurity: Tackling challenges related to data exchange and security: An edge-computing-enabled secure and efficient data exchange architecture for the energy internet," IEEE Consumer Electronics Magazine, vol. 8, no. 2, pp. 61-65, 2019.

[23] M. Z. Khan, S. Harous, S. U. Hassan, M. U. Ghani Khan, R. Iqbal, and S. Mumtaz, "Deep unified model for face recognition based on convolution neural network and edge computing," IEEE Access, vol. 7, pp. 72622-72633, 2019.

[24] A. Mahmood, A. Ahmed, M. Naeem, and Y. Hong, "Partial offloading in energy harvested mobile edge computing: A direct search approach," IEEE Access, vol. 8, pp. 36 757-36763, 2020.

[25] M. V. Barbera, S. Kosta, A. Mei, and J. Stefa, "To offload or not to offload? the bandwidth and energy costs of mobile cloud computing," in 2013 Proceedings Ieee Infocom. IEEE, 2013, pp. 1285-1293.

[26] Y. Liu, M. J. Lee, and Y. Zheng, "Adaptive multi-resource allocation for cloudlet-based mobile cloud computing system," IEEE Transactions on Mobile Computing, vol. 15, no. 10, pp. 2398-2410, 2015.

[27] M. Chen, Y. Hao, Y. Li, C.-F. Lai, and D. Wu, "On the computation offloading at ad hoc cloudlet: architecture and service modes," IEEE Communications Magazine, vol. 53, no. 6, pp. 18-24, 2015.

[28] L. Tong, Y. Li, and W. Gao, "A hierarchical edge cloud architecture for mobile computing," in IEEE INFOCOM 2016-The 35th Annual IEEE International Conference on Computer Communications. IEEE, 2016, pp. 1-9.

[29] Y. Sun, S. Zhou, and J. Xu, "Emm: Energy-aware mobility management for mobile edge computing in ultra dense networks," IEEE Journal on Selected Areas in Communications, vol. 35, no. 11, pp. 2637-2646, 2017.

[30] Y. Xiao and M. Krunz, "Qoe and power efficiency tradeoff for fog computing networks with fog node cooperation," in IEEE INFOCOM 2017-IEEE Conference on Computer Communications. IEEE, 2017, pp. $1-9$.

[31] M. Chen and Y. Hao, "Task offloading for mobile edge computing in software defined ultra-dense network," IEEE Journal on Selected Areas in Communications, vol. 36, no. 3, pp. 587-597, 2018.

[32] D.-J. Deng, S.-Y. Lien, C.-C. Lin, S.-C. Hung, and W.-B. Chen, "Latency control in software-defined mobile-edge vehicular networking," IEEE Communications Magazine, vol. 55, no. 8, pp. 87-93, 2017.

[33] L. Huang, S. Bi, and Y. J. Zhang, "Deep reinforcement learning for online computation offloading in wireless powered mobile-edge computing networks," IEEE Transactions on Mobile Computing, 2019.

[34] G. Liu, Y. Xu, Z. He, Y. Rao, J. Xia, and L. Fan, "Deep learningbased channel prediction for edge computing networks toward intelligent connected vehicles," IEEE Access, vol. 7, pp. 114 487-114 495, 2019.

[35] S. Lai, J. Xia, D. Zou, and L. Fan, "Intelligent secure communication for cognitive networks with multiple primary transmit power," IEEE Access, vol. 8, pp. 37343-37351, 2020.

[36] J. Xia, K. He, W. Xu, S. Zhang, L. Fan, and G. K. Karagiannidis, "A mimo detector with deep learning in the presence of correlated interference," IEEE Transactions on Vehicular Technology, vol. 69, no. 4, pp. 4492-4497, 2020.

[37] Y. Xu, J. Xia, H. Wu, and L. Fan, "Q-learning based physical-layer secure game against multiagent attacks," IEEE Access, vol. 7, pp. 49212-49 222, 2019.

[38] J. Xia, Y. Xu, D. Deng, Q. Zhou, and L. Fan, "Intelligent secure communication for internet of things with statistical channel state information of attacker," IEEE Access, vol. 7, pp. 144481-144488, 2019.

[39] S. Le Digabel, "Algorithm 909: Nomad: Nonlinear optimization with the mads algorithm," ACM Transactions on Mathematical Software (TOMS), vol. 37, no. 4, pp. 1-15, 2011.

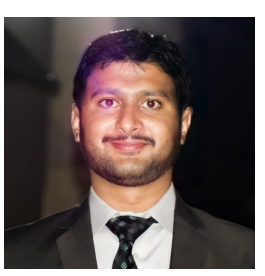

Asad Mahmood received his Master degrees in Electrical Engineering from the Department of Electrical Computer Engineering, COMSATS University Islamabad, Wah Campus, Pakistan. Currently working as a Research Assistant with Dr. Yue Hong at College of Mechatronics and Control Engineering, Shenzhen University, Shenzhen, China. His research interest include resource allocation in wireless communication, Mobile Edge Computing, Machine learning, Evolutionary Algorithm, and Classic Optimization

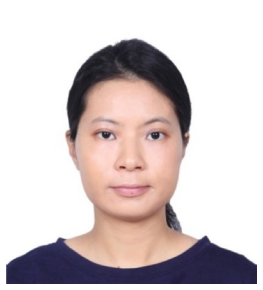

Yue Hong received the Ph.D. degree in engineering science from Uppsala University, Uppsala, Sweden, in 2017. She is currently an Assistant Professor at College of Mechatronics and Control Engineering, Shenzhen University, Shenzhen, China. Her research interest mainly focuses on renewable energy generation especially wave energy conversion, includes wave energy resource assessment, electrical control on wave energy conversion, and hybrid system management.

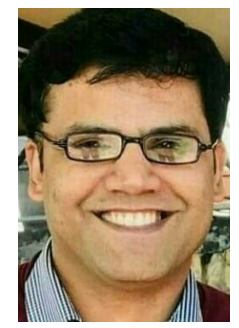

Muhammad Khurram Ehsan Muhammad Khurram Ehsan is currently working as Assistant Professor, Faculty of Engineering, Bahria University, Pakistan since Sep, 2017. He did his PhD in Engineering with specialization in statistical signal processing and MS in Electrical Communication Engineering from University of Kassel, Germany in 2016 and 2010 respectively. As a reviewer, he is closely working with Elsevier journal of Network and Computer Applications and also with IEEE systems Journal. His research interest includes statistical modeling, data analysis and cognitive radio enabled systems including wireless sensor networks and Internet of things 


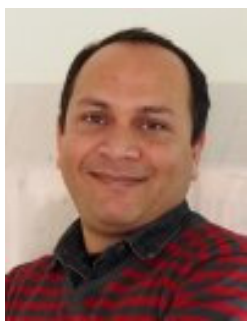

Shahid Mumtaz (M'13-SM'16) received the M.Sc.degree in electrical and electronic engineering from the Blekinge Institute of Technology, Karlskrona,Sweden, in 2006, and the Ph.D. degree in electrical and electronic engineering from the University of Aveiro, Aveiro, Portugal, in 2011. He was a Research Intern with Ericsson, Stockholm, Sweden, and Huawei Research Laboratory, Karlskrona, Sweden, in 2005. He has more than thirteen years of wireless industry experience and is currently a Senior Research Scientist and the Technical Manager with the Instituto de Telecommuninições Aveiro, Aveiro, Portugal. $\mathrm{He}$ has authored several conferences, journals, and books publications. His current research interests include field of architectural enhancements to 3GPP networks (i.e., LTE-A user plan and control plan protocol stack, NAS, and EPC), 5G-related technologies, green communications, cognitive radio,cooperative networking, radio resource management, cross-layer design, backhaul/fronthaul, heterogeneous networks, machine-to-machine and deviceto-device communication, and baseband digital signal processing 